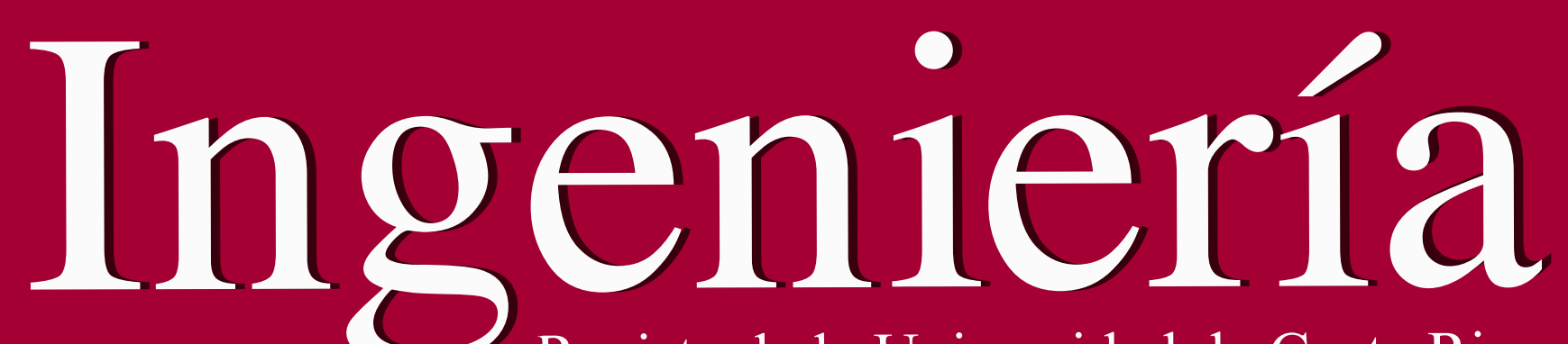

Revista de la Universidad de Costa Rica JULIO / DICIEMBRE 2020 - VOLUMEN 30 (2)

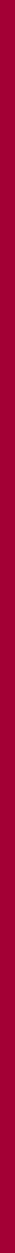




\title{
Algoritmo de optimización para sistema de gestión eléctrica inteligente
}

\section{Optimization algorithm for intelligent electrical energy management system}

\author{
José David Murillo Rodríguez. \\ Universidad de Costa Rica, San José, Costa Rica. \\ jose.murillorodriguez@ucr.ac.cr \\ ORCID: https://orcid.org/0000-0003-3448-2346
}

Recibido: 6 de febrero 2020 Aceptado: 12 de mayo 2020

\section{Resumen}

Mediante un algoritmo de optimización, se muestra el comportamiento de una gestión eléctrica inteligente basado en la combinación multiagente de almacenamiento y energía alternativa. El algoritmo de control se concibe a través de una optimización del flujo energético de una residencia. Con datos reales de consumo y generación prevista, la optimización fija un límite de ahorro del $93 \%$ respecto a un sistema tradicional sin gestión eléctrica. De acuerdo con el flujo energético y bajo las mismas condiciones de optimización, se comprueba el funcionamiento del algoritmo, el cual permite acercarse al límite y lograr un $86 \%$ de ahorro.

Palabras clave:

Ingeniería eléctrica, Fuente de energía renovable, Casa, Ahorro, Consumo de energía

\begin{abstract}
Through an optimization algorithm, the behavior of intelligent electrical energy management based on the multiagent combination of storage and alternative electric energy is shown. The control algorithm is conceived altogether with the optimization and the energy flow of a residence. With real consumption and expected generation data, the optimization sets a savings limit of $93 \%$ compared to a traditional system without electrical energy management. According to the energy flow and under the same optimization conditions, the operation of the algorithm is verified, and the results approach the limit and achieve savings of $86 \%$.
\end{abstract}

Keywords:

Electrical engineering, Renewable energy sources, Houses, Savings, Energy consumption 


\section{INTRODUCCIÓN}

Actualmente existe un amplio desarrollo tecnológico de nuevas fuentes de energía alternativa renovable, más accesibles y adecuadas para el sector residencial. Celdas solares fotovoltaicas (PV, del inglés Photovoltaics), celdas de hidrógeno, sistemas de micro-generación eólica y otros son casos de fuentes alternativas asequibles en las residencias (Haider, See \& Elmenreich, 2016). Teniendo en cuenta las carencias y defectos que posee la tecnología actual, se piensa en la próxima generación de redes de sistemas eléctricos Smart-grid, donde la comunicación y el manejo de datos son esenciales para este nuevo paradigma (Farhangi, 2010; Ipakchi \& Albuyeh, 2009). La Tabla 1 contrasta algunos rasgos importantes entre esquemas convencionales y Smart-grid.

Tabla 1. Comparación entre Smart-Grid y esquema convencional. Adaptado de Farhangi (2010)

\begin{tabular}{cc}
\hline Red Existente & Smart-grid \\
\hline Una vía de comunicación & Doble vía de comunicación \\
Generación centralizada & Generación distribuida \\
Topología jerárquica & Topología de malla \\
Pocos sensores & Sensores predominantes \\
Restauración manual & Autorreparación \\
Fallas y apagones & Adaptativa y aislante \\
Chequeo y control manual & Chequeo y control remoto \\
\hline
\end{tabular}

\subsection{Sistemas de gestión energética residencial: NanoGrid}

Adaptando el concepto de Smart-grid al entorno residencial, el resultado es una NanoGrid. Una NanoGrid cuenta con las características importantes de Smart-grid a bajas potencias (Amjad et al., 2015).

Un esquema típico de NanoGrid se observa en la Figura 1-a.

El corazón de la NanoGrid residencial es el sistema de control energético o REMS (del inglés Residential Energy Management System) (Refaat \& Haitham, 2015). De acuerdo con las condiciones de la casa, el usuario y el entorno, el REMS tomará decisiones para el flujo energético. Los REMS se distinguen por esquemas de control distribuido y multiagente (Pipattanasomporn \& Feroze, 2009). La Figura 1-b representa una arquitectura típica de REMS asociada a la NanoGrid descrita anteriormente.

En general, el REMS consiste en el agente FER, encargado de supervisar la generación de la fuente renovable. La alimentación proveniente de la empresa distribuidora es controlada por el agente FEED y el agente LOAD supervisa la demanda de las cargas. El agente BAT se encarga 
del almacenamiento energético. Estos cuatro agentes se comunican entre sí y no dependen de los demás. También existe un agente con conectividad DAT, que almacena y comparte información de acuerdo a las necesidades del REMS.

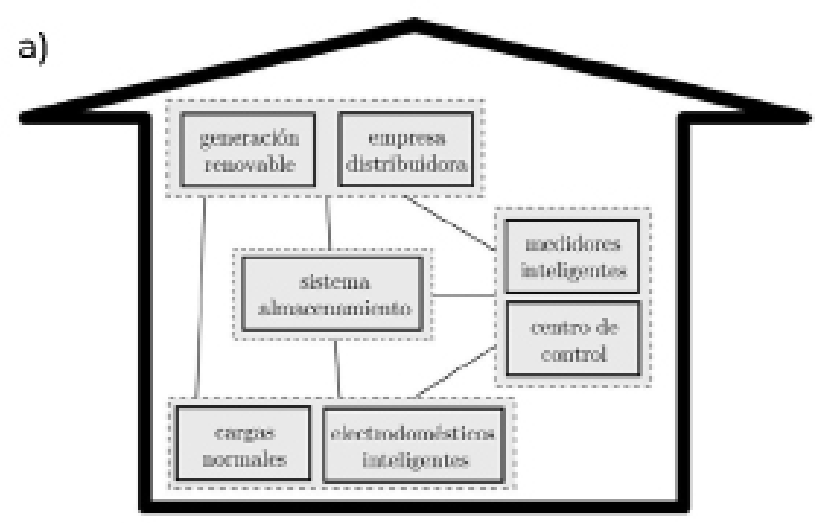

b)

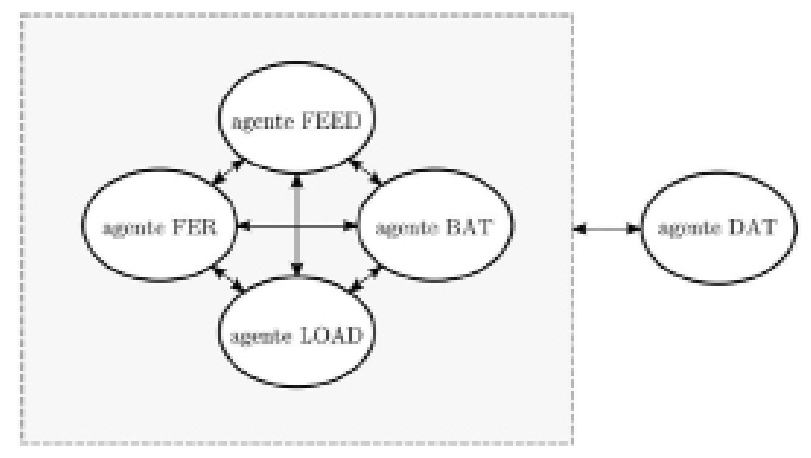

Figura 1. Esquema general de NanoGrid residencial a) y su REMS asociado b). Fuente: Elaboración propia

\section{METODOLOGÍA}

El desarrollo del trabajo incluyó los pasos y procedimientos mostrados en la tabla 2, clasificados por subtema.

Tabla 2. Metodología propuesta

\begin{tabular}{cc}
\hline Subtema & Metodología \\
\hline Especificación de problema a resolver & Investigación sobre arquitecturas de NanoGrids y REMS \\
& Definición de estructura de NanoGrid \\
Optimización y algoritmo & Esquematización y definición de arquitectura de REMS \\
\hline Validación & Desarrollo de optimización de acuerdo a NanoGrid propuesta \\
\hline
\end{tabular}




\section{OPTIMIZACIÓN Y DESARROLLO DEL ALGORITMO}

Para el REMS, se propone un sistema multiagente que gestione la energía de forma optimizada. Los cinco tipos agentes definidos conforman la propuesta del sistema y se asignan a cada elemento residencial. Como se trata de un sistema modular, se pueden agregar tantos agentes como elementos se requiera, sin embargo, para este caso se propone un caso general donde existe un agente para cada tipo.

\subsection{Gestión Energética}

La gestión inteligente de la energía se lleva a cabo al tratar de minimizar el costo energético. La idea principal es minimizar el consumo de energía proveniente de la red de distribución. Si se divide el día en 24 horas, en cada hora $i$ el flujo de potencia residencial está dado por la ecuación 1.

$$
P_{i}^{r e d}=P_{i}^{c o n}-P_{i}^{g e n}+P_{i}^{c b a t}-P_{i}^{d b a t}
$$

$P_{i}^{r e d}$ representa la potencia demandada por el agente FEED. $P_{i}^{\text {gen }}$ es la potencia total generada por el agente FER y $P_{i}^{c b a t}$ es la potencia consumida por el agente LOAD. Además, se tiene $P_{i}^{c b a t}$ y $P_{i}^{d b a t}$ que corresponden a la potencia de carga y descarga del agente BAT. Por simplicidad, BAT se modela con dos potencias y sus valores están delimitados por las características del agente, que son mutuamente excluyentes ya que la batería no se puede descargar y cargar al mismo tiempo. Con una tarifa horaria $\bar{\lambda}_{i}$, el costo diario $F_{d}$ ante la empresa distribuidora está dado por 2 .

$$
F_{d}=\sum_{i}^{24} \lambda_{i} P_{i}^{r e d}=\lambda_{i}\left(P_{i}^{c o n}-P_{i}^{g e n}+P_{i}^{c b a t}-P_{i}^{d b a t}\right)
$$

La cantidad $F_{d}$ es la que se debe minimizar controlando únicamente $P_{i}^{\text {cbat }}$ y $P_{i}^{d b a t}$.

\subsection{Problema de optimización}

De la ecuación 2 se tiene información de la potencia consumida, la potencia generada y la tarifa horaria. La información de cada uno de estos aspectos se clasifica como "datos de entrada" del problema. Datos reales de potencia consumida, promedio y diaria en hogares se obtienen del Uvecase (Unidad de verificación de la calidad del suministro eléctrico) de la Universidad de Costa Rica (2017). La fuente renovable se modela como un panel fotovoltaico de $1 \mathrm{~kW}$ y los datos promedio de producción para San José se adquieren de Rojas y Valverde (2014). La tarifa horaria se obtiene de la CNFL (2016). El problema se define como una optimización lineal con restricciones no lineales. En este caso, se deben encontrar los valores $P_{i}^{r e d}, P_{i}^{c b a t}$ y $P_{i}^{d b a t}$ que minimicen $F_{d}$. Estos valores se agrupan en un vector $\bar{x}=\left[P_{1}^{\text {red }}, P_{2}^{\text {red }}, \ldots, P_{24}^{\text {red }}, P_{1}^{\text {cbat }}, P_{2}^{\text {cbat }}, \ldots, P_{24}^{\text {cbat }}, P_{1}^{\text {dbat }}, P_{2}^{\text {dbat }}, \ldots, P_{24}^{\text {dbat }}\right]$.

En este caso, las restricciones recaen en la mutual exclusión de la carga y descarga de BAT $\left(P_{i}^{\text {cbat }}\right.$ x $\left.P_{i}^{d b a t}=0\right)$, además que estas no pueden ser negativas $\left(P_{i}^{c b a t} \geq 0\right),\left(P_{i}^{d b a t} \geq 0\right)$. Por simplicidad se 
considera que no se puede inyectar energía a la red $\left(P_{i}^{\text {red }} \geq 0\right)$ y se establece la limitante de un ciclo único diario de carga y descarga de la batería $\left(P_{1}^{c b a t}+P_{2}^{c b a t}+\ldots+P_{24}{ }^{c b a t}-P_{1}^{d b a t}-P_{c}^{c b a t}-P_{24}{ }^{c b a t} \ldots=0\right)$.

Con ayuda de "fmincon" de MATLAB, se resuelve la optimización $\bar{x}_{\text {min }} \sum_{i=0}^{24} \lambda_{i} P_{i}^{\text {red }}$ y se encuentran los valores que minimizan el costo de energía comprada. El resultado se muestra en la Figura 2-a incluyendo la fuente renovable y en 2-b excluyéndola.

a)

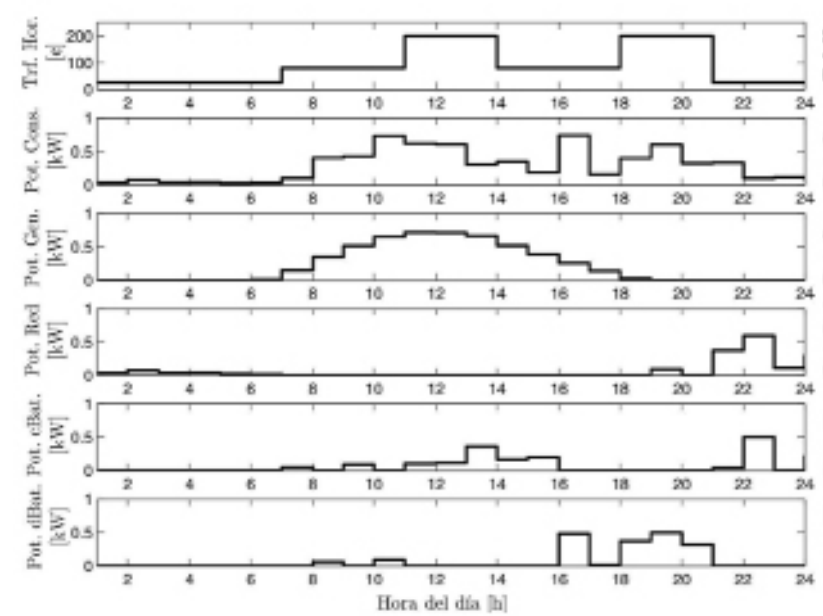

b)

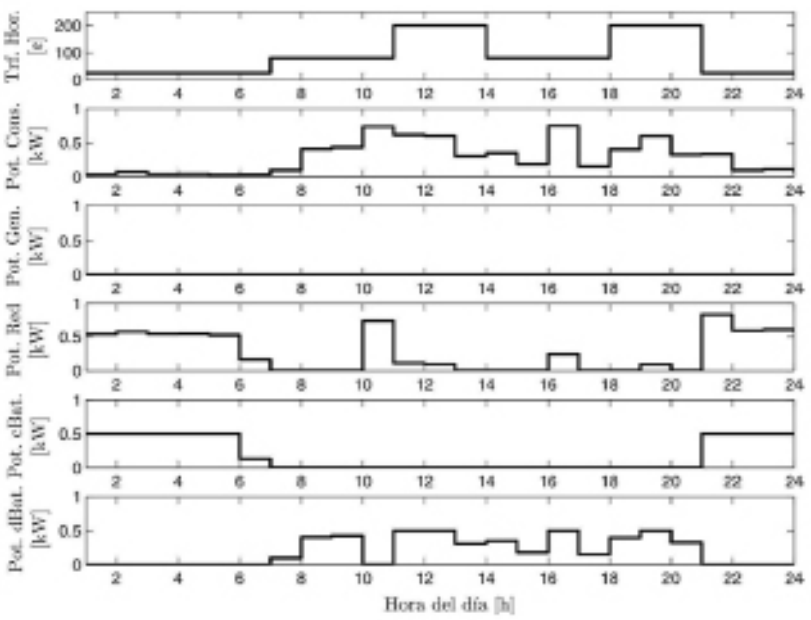

Figura 2. Resultado para el problema de optimización a) con sistema fotovoltaico y b) sin sistema fotovoltaico. Fuente: Elaboración propia

\subsection{Algoritmo optimizado}

Con los resultados prometedores de la optimización, se crea un algoritmo que sea capaz de obtener resultados cercanos. Como las decisiones únicamente se toman en la batería (agente BAT) y en el alimentador principal residencial (agente FEED), se crean dos variantes del algoritmo. El agente FEED debe controlar el alimentador principal de acuerdo a las condiciones de FER, LOAD y BAT. El diagrama de flujo de la Figura 3-a representa el algoritmo propuesto para el agente FEED. En la Figura 3-b se muestra el diagrama de flujo correspondiente a la variante del algoritmo para el agente BAT. En este caso se tienen tres estados para la batería. BAT CHARGE implica que se habilita solamente la carga de la batería. BAT DSCHRGE implica habilitar la descarga y BAT OFF corresponde a inhabilitar tanto la carga como la descarga.

En la Figura 4-a, se observa en azul punteado los valores óptimos obtenidos al ejecutar el algoritmo con los mismos datos preliminares que la optimización, mientras que en la Figura 4-b se considera el caso de no tener un PV. 
a)

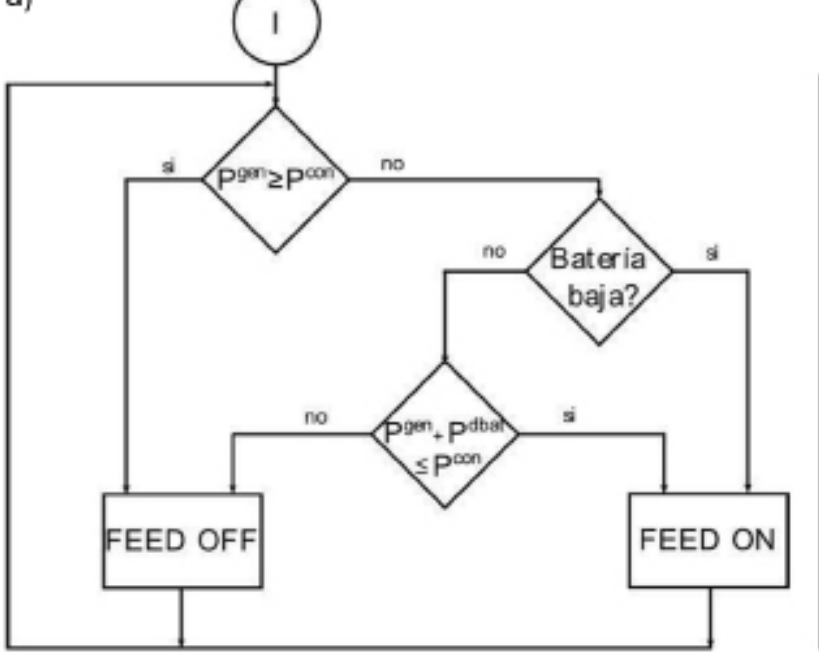

b)

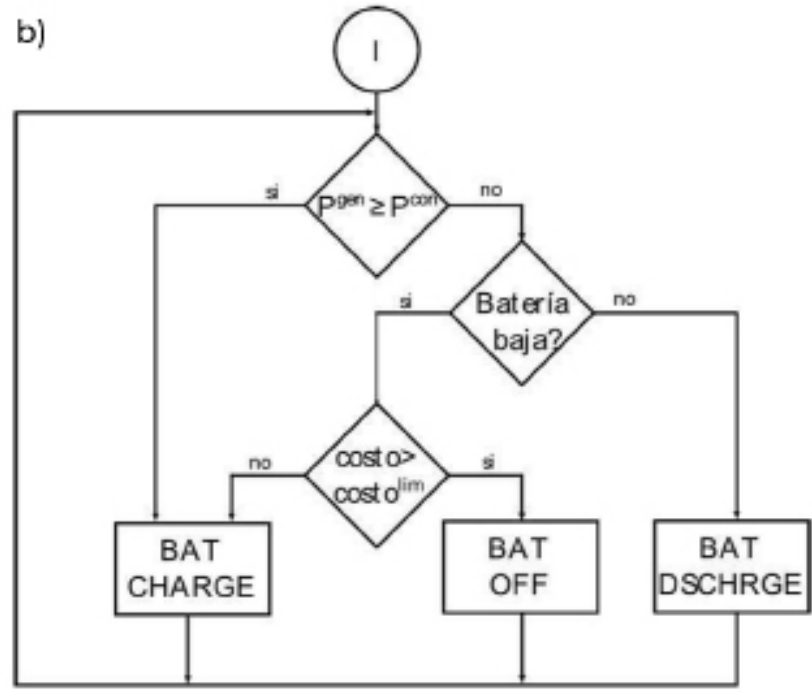

Figura 3. Algoritmo obtenido a través de la optimización, variante a) para agente RED y b) para agente BAT.

Fuente: Elaboración propia
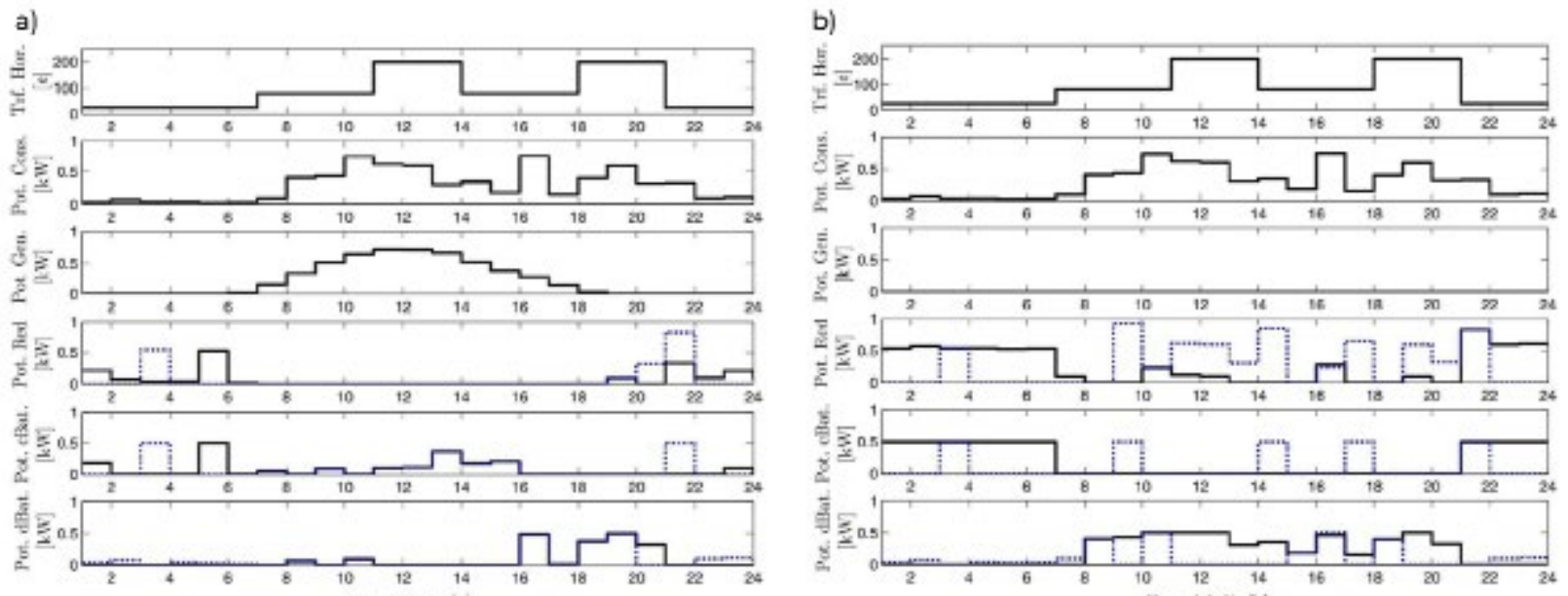

Figura 4. Comparación entre algoritmo y resultado de optimización a) con sistema fotovoltaico y b) sin sistema fotovoltaico. Fuente: Elaboración propia

\section{CONCLUSIONES}

En la Tabla 3 se muestran los resultados obtenidos mediante la optimización y el algoritmo. De las comparaciones resultantes, se nota que el sistema hace lo posible para minimizar el costo energético proveniente de la empresa distribuidora. El ahorro por parte del algoritmo está muy cerca del límite teórico obtenido con la optimización.

Para un análisis posterior se puede extender la optimización incorporando la entrega de energía a la red y así evaluar el algoritmo obtenido e incluso crear un nuevo algoritmo bajo estas condiciones. 
Tabla 3. Resultados económicos de optimización de costo energético de la empresa distribuidora

\begin{tabular}{ccc}
\hline Caso & Costo mensual promedio [đ] & Ahoro [\%] \\
\hline Sin optimización & 25032 & 0 \\
Optimización con PV & 1783 & 92.88 \\
Optimización sin PV & 8302 & 66.83 \\
Algoritmo optimizado con PV & 3531 & 85.89 \\
Algoritmo optimizado sin PV & 22602 & 9.71 \\
\hline
\end{tabular}

Nota: Basado en tarifas eléctricas residenciales CNFL.(Octubre 2016). San José, Costa Rica

\section{SIMBOLOGÍA}

BAT

BAT CHARGE

BAT DSCHRGE BAT OFF

CNFL

DAT

$F_{d}$

FEED

FER

FEED ON FEED OFF

$\bar{\lambda}_{i} \wedge$

LOAD

NanoGrid

$P_{i}^{\text {red }}$

$P^{\text {gen }}$

$P^{c o n}$

$P_{i}^{d b a t}$

$P_{i}^{c b a t}$

$P_{i}^{\text {red }}$

PV

REMS agente encargado del almacenamiento eléctrico en la NanoGrid.

estado de carga del agente BAT.

estado de descarga del agente BAT.

estado de apagado del agente BAT.

Compañía Nacional de Fuerza y Luz.

agente que almacena información y detalles de lo que está sucediendo en la NanoGrid.

Costo de energía diario en la NanoGrid.

agente que representa la alimentación proveniente de la empresa distribuidora en la NanoGrid.

agente encargado de supervisar la generación, estado, habilitación y

disponibilidad de la fuente renovable en la NanoGrid.

estado de encendido del agente FEED.

estado de apagado del agente FEED.

tarifa residencial horaria de la CNFL.

agente que supervisa la demanda de las cargas eléctricas en la NanoGrid.

Smart-grid residencial.

representa la potencia demandada por el agente FEED.

es la potencia total generada por el agente FER.

es la potencia consumida por el agente LOAD.

potencia de descarga del agente BAT.

potencia de carga del agente BAT.

potencia proveniente de la red de distribución eléctrica en la hora del día i. del inglés Photovoltaics, sistema Fotovoltaico.

del inglés Residential Energy Management System, Sistema de Administración 


\section{Energético Residencial.}

Smart-grid Red eléctrica inteligente.

Uvecase

Unidad de verificación de la calidad del suministro eléctrico de la Universidad de Costa Rica

$\bar{x}$

Arreglo matemático de las potencias de cada uno de los agentes de la NanoGrid.

\section{REFERENCIAS}

Amjad, A., Monsef, H., Ashkan, R., Guerrero, J., y Vasquez, J. (Julio, 2015). Optimized energy management of a single-house residential micro-grid with automated demand response. En: IEEE Eindhoven PowerTech. Conferencia realizada en Eindhoven, Países Bajos.

CNFL. (Octubre, 2016). Tarifa Residencial Horaria. Compañía Nacional de Fuerza y Luz. Recuperado de https://www.cnfl.go.cr/index.php/tarifas-vigentes-r.

Farhangi, H. (Diciembre, 2010). The path of the smart grid. IEEE Power Energy Magazine, 8(1), 18-28.

Haider, H., See, O., y Elmenreich, W. (2016, Junio). A review of residential demand response of smart grid. Renew Sustain Energy Rev, 59, 166-178.

Ipakchi, A. y Albuyeh, F. (Febrero, 2009). Grid of the future. IEEE Power and Energy Magazine, 7(2), 52-62.

Pipattanasomporn, M. y Feroze, H. (Marzo, 2009). Multi-agent systems in a distributed smart grid: Design and implementation. En: IEEE/PES Power Systems Conference. Conferencia realizada en Seattle, Estados Unidos.

Refaat, S. y Haitham, A. (Septimbre, 2015). Implementation of smart residential energy management system for smart grid. En: IEEE Energy Conversion Congress and Exposition. Conferencia realizada en Montreal, Canadá.

Rojas, J. y Valverde, G. (2014). Reporte SolarGIS (Informe N 01). San José, Costa Rica: Escuela de Ingeniería Eléctrica, Universidad de Costa Rica.

Uvecase. (2017). Unidad Verificadora de la Calidad del Suministro Eléctrico. San José, Costa Rica: Universidad de Costa Rica. Recuperado de http://celeq.ucr.ac.cr/servicios/uvecase 